\title{
Uma Revisão Sistemática sobre as Abordagens Ubíquas para Recomendação Educacional: Estariam Elas se Tornando Adaptativas?
}

\author{
Guilherme M. Machado1, Vinícius Maran ${ }^{1,4}$, Isabela Gasparini², Ana M. Pernas ${ }^{3}$, \\ José Palazzo M. de Oliveira ${ }^{1}$ \\ ${ }^{1}$ Instituto de Informática - UFRGS - Porto Alegre, RS - Brasil \\ ${ }^{2}$ Departmento de Ciência da Computação - UDESC - Joinville, SC - Brasil \\ ${ }^{3}$ Centro de Desenvolvimento Tecnológico - UFPel - Pelotas, RS - Brasil \\ ${ }^{4}$ Coordenadoria Acadêmica - UFSM - Cachoeira do Sul, RS - Brasil \\ $\{$ g.medeiros, vmaran, palazzo\}einf.ufrgs.br, \\ isabela.gasparini@udesc.br, anamarilzalinf.ufpel.edu.br
}

\begin{abstract}
Recommender and Adaptive systems have their focus in providing items and services to the user. The recommender leaves up to the user the decision of using or not the item or service and the adaptive enforcing a behavior. By such characteristics, their goal in the educational domain often are similar, if not the same. Despite such similarities, there are few papers in the literature trying to investigate the possibility of integration between the two domains. The goal of this paper is to investigate if the latest recommender approaches for educational ubiquitous environments are also trying to integrate adaptive strategies to their recommendations. The results of our systematic mapping opens new research possibilities. Our findings pointed that there are no papers investigating the utilization of a hybrid approach that leverages contribution to both recommender and adaptive systems.
\end{abstract}

Resumo. Sistemas de recomendação e adaptativos tem como foco oferecer itens e serviços a usuários. Sistemas de recomendação geralmente deixam a cargo do usuário a escolha de utilizar ou não utilizar um item recomendado, enquanto sistemas adaptativos forçam um comportamento. Aplicados à área da educação, estes sistemas possuem objetivos similares. Apesar disso, poucos trabalhos têm investigado a possibilidade de integração entre estes tipos de sistema. Neste cenário, este artigo tem por objetivo investigar as últimas abordagens de recomendação para ambientes educacionais ubíquos e verificar se existe alguma forma de integração entre as abordagens adaptativas e as de recomendação. Os resultados do mapeamento mostraram novas possibilidades de pesquisa na área, já que não foi encontrada uma solução, dentre as pesquisadas, que utilize uma abordagem híbrida que contemple as contribuições oferecidas por métodos de recomendação e de adaptação.

\section{Introdução}

Sistemas hipermídia adaptativos (ou simplesmente sistemas adaptativos) são sistemas que refletem algumas características dos alunos no modelo do usuário e aplicam este modelo para adaptar vários aspectos visíveis do sistema [Gasparini et al. 2009a]. Técnicas de 
adaptação hipermídia têm sido utilizadas com sucesso já algum tempo em sistemas $e$ learning com objetivo de prover navegação, apresentação e conteúdos educacionais adaptados às características individuais de cada aluno [Brusilovsky e $\mathrm{Su}$ 2002], transpondo assim as limitações apresentadas por antigos sistemas e-learning do tipo "one size fits all" [Brusilovsky 2001], onde o mesmo conteúdo era apresentado da mesma maneira a todos os usuários. Com uma proposta similar, os sistemas de recomendação têm por objetivo sugerir itens de interesse aos usuários [Ricci et al. 2011]. Alguns sistemas de recomendação já são utilizados para sugerir objetos de aprendizagem aos usuários [Bouzeghoub et al. 2009; Gasparini et al. 2009; Medeiros Machado e De Oliveira 2014].

Uma informação que ambos os sistemas levam em consideração ao recomendar ou adaptar conteúdo ao usuário é a de contexto. A percepção do contexto do usuário permite que tais sistemas sejam aptos a recomendar ou adaptar elementos não apenas restritos ao sistema de informação, mas presentes no ambiente que o usuário está inserido. Essa percepção é importante, pois possibilita que o aprendizado se torne ubíquo, ou seja, que este aconteça associada a um estudante sem que o mesmo tenha consciência do processo (aprendizagem ubíqua) [Jones e Jo 2004].

O presente trabalho tem como objetivo realizar um mapeamento sistemático referente à área de aprendizagem ubíqua, mais especificamente sobre o estado da arte das técnicas de recomendação e adaptação utilizadas neste domínio e como elas estão relacionadas. Para isso, definiu-se uma questão de pesquisa, apresentada na próxima seção, que leva em consideração quatro áreas principais relacionadas aos sistemas educacionais, são elas: (i) sensibilidade ao contexto; (ii) sistemas de recomendação; (iii) sistemas adaptativos; e (iv) ubiquidade. O restante deste artigo está estruturado da seguinte maneira. $\mathrm{Na}$ Seção 2 é apresentado o processo do mapeamento sistemático. Na Seção 3 são introduzidos conceitos sobre Computação Ubíqua e Sensibilidade ao Contexto, Sistemas de Recomendação e Sistemas Adaptativos. Na Seção 4, os trabalhos são categorizados e analisados para responder à questão de pesquisa. Finalmente as conclusões são apresentadas na Seção 5 .

\section{Revisão Sistemática de Literatura}

O estudo de revisão sistemática é um método de pesquisa que fornece guias para conduzir revisões de literatura. Ele permite que evidências sobre um determinado domínio sejam reveladas em um alto nível de granularidade e que sejam identificados trabalhos agrupados de acordo com uma série de características, definidas pelos revisores [Kitchenham e Charters 2007]. A revisão apresentada neste trabalho foi conduzida pela metodologia apresentada por Petersen [Petersen et al. 2008]. As principais etapas da revisão sistemática são: (i) Definição de questões de pesquisa; (ii) Busca por trabalhos relevantes (estudos validados que apresentam contribuições significativas para a área); (iii) Seleção e filtragem de trabalhos (por critérios de inclusão e exclusão), e (iv) Extração de dados e mapeamento.

A questão de pesquisa definida neste trabalho é: "Existe alguma abordagem de recomendação que pode ser aplicada em ambientes ubíquos tradicionais e ambientes ubíquos educacionais, que seja sensivel ao contexto e adaptativa?". Sensibilidade ao contexto no escopo deste trabalho está relacionada à habilidade de sistemas em gerenciar informações de contexto. Adaptação se refere a capacidade de sistemas se adaptarem a pelo menos uma das características definidas por [Brusilovsky e Su 2002] ou adaptarem 
o ambiente de alguma forma. Ambientes ubíquos são ambientes dotados de computadores em que a interação entre a computação e o usuário ocorre de maneira transparente [Weiser 1991]. O principal objetivo é identificar como tais áreas do conhecimento estão relacionadas entre si, e identificar lacunas e oportunidades de pesquisa. A primeira etapa da revisão trata da definição do argumento de busca (string de busca). Para defini-lo, foram identificadas as palavras primárias na questão de pesquisa. Estas palavras estão relacionadas com o domínio-foco da revisão sistemática. Após esta identificação, as palavras foram submetidas a um dicionário de sinônimos para adicionar possíveis sinônimos e variações das palavras à string de busca. A string utilizada para procura de trabalhos em diversos mecanismos de busca foi:

(approach OR method* OR model) AND (recommend*) AND (pervasi* OR ubiquitous) AND (aware OR sensi*) AND (context OR situation) AND (adapt*)

Após definir a string, a pesquisa por trabalhos foi realizada em quatro mecanismos de busca: ACM Digital Library, IEEE Xplore Digital Library, Springer Link e Elsevier. Os critérios de inclusão (I) e exclusão (E) apresentados a seguir foram utilizados na seleção dos artigos para a análise.

I1. Artigos científicos publicados em eventos (por exemplo, conferências, congressos, simpósios, workshops, entre outros) ou periódicos relacionados à área de ciência da computação;

I2. Estudos inéditos;

I3. Artigos completos (quatro ou mais páginas);

I4. Artigos publicados entre 2010 e 2014;

E1. Artigos publicados em outras línguas exceto inglês;

E2. Artigos que não utilizaram ao menos três dos principais tópicos (computação ubíqua, sensibilidade ao contexto, sistemas adaptativos ou sistemas de recomendação).

A busca inicial, utilizando a string de busca, retornou 419 artigos (Tabela 1). Após a leitura do título, palavras-chave e abstract dos mesmos, foi realizada uma filtragem ( $\mathbf{1}^{\mathbf{a}}$ Filt.) para obter os artigos considerados como diretamente relacionados à questão de pesquisa. Como resultado, foram obtidos 154 artigos após a realização da primeira filtragem.

Tabela 1. Artigos obtidos após a realização das filtragens

\begin{tabular}{|l|l|l|l|}
\hline Motor de Busca & Recuperados & $\mathbf{1}^{\text {a }}$ Filt. & $\mathbf{2}^{\text {a }}$ Filt. \\
\hline ACM Digital Library & 214 & 75 & 28 \\
\hline Elsevier & 34 & 22 & 10 \\
\hline IEEE Xplore Digital & 82 & 18 & 1 \\
\hline Springer Link & 89 & 39 & 4 \\
\hline Total & 419 & 154 & 43 \\
\hline
\end{tabular}

Cada artigo retornado foi analisado com base no título, palavras-chave, resumo, conclusão e em alguns casos, as seções referentes as propostas dos trabalhos. Nesta etapa, o foco não foi analisar tecnologias e técnicas de baixo nível, e sim ter uma visão de alto nível sobre como as propostas trabalharam com os problemas de pesquisa apontados. Cada artigo foi classificado de acordo com as quatro áreas relacionadas com a pesquisa de mapeamento sistemático. Como o foco é identificar lacunas e oportunidades de pesquisa nestas áreas, foram selecionados apenas artigos relacionados à pelo menos três das quatro áreas de pesquisa ( $2^{\mathbf{a}}$ Filt.). Foram então obtidos 43 artigos, que foram clusterizados, lidos e analisados. Dentre os artigos, 43 artigos analisados apresentam soluções relacionadas a sensibilidade ao contexto, 31 possuem propostas que envolvem técnicas de recomendação, 41 são relacionados a área de computação ubíqua e 17 destes apresentam 
alguma abordagem adaptativa. Mais explicações sobre o processo de análise são apresentadas na Seção 4. A lista de artigos analisados está disponível em: http://bit.ly/1VFm4Nb.

\section{Fundamentação}

A computação ubíqua é um paradigma que utiliza ambientes ricos em recursos computacionais e de comunicação de forma integrada aos objetos do dia a dia, utilizados por pessoas. Assim, a computação pode auxiliar as pessoas na realização de suas tarefas diárias de forma transparente, mesmo sem que os usuários percebam a computação envolvida nestes processos. Para criar estes ambientes, tecnologias, modelos e dispositivos têm sido propostos e testados em diversas áreas. Entretanto, a implementação plena de cenários ubíquos como os descritos por [Weiser 1991] continuam sendo um desafio, mesmo diante dos avanços significativos na área.

Contexto, segundo [Dey 2001], é definido como "qualquer informação que pode ser utilizada para caracterizar a situação de entidades (pessoa, lugar ou objeto) que são consideradas relevantes para a interação entre o usuário e a aplicação". Em definições mais recentes [Makris et al. 2013], contexto é definido como um conhecimento medido e inferido a partir de fluxos contínuos de dados que descrevem o estado de entidades.

Uma série de trabalhos em sensibilidade ao contexto têm seu foco em apresentar formas de modelagem e representação de contexto em sistemas computacionais. As formas mais utilizadas de modelagem são: Pares chave-valor (Key-value pairs - KV), orientação a objeto (OO), baseados em lógica (Logic based - LB), modelos gráficos (Graphic models - GM) e ontologias (O) [Bettini et al. 2010] [Makris et al. 2013]. Algumas propostas utilizam mais de uma forma de modelagem, resultando em abordagens híbridas [Riboni e Bettini 2012] [Maran et al. 2014].

Para prover informações sobre o estado de entidades nesses sistemas, diferentes níveis de contexto são relevantes. A definição mais utilizada é de [Bettini et al. 2010], onde os níveis de contexto são definidos como: (i) Baixo Nível (LL): Sensores coletam informações sobre o ambiente, (i) Nível Intermediário (IL): Dados de contexto são medidos ou inferidos, agregando informações em um nível mais alto de abstração se comparado ao contexto de baixo nível, e (iii) Alto Nível (HL): Informações de contexto são agregadas através da definição de relações semânticas entre as informações para ligar informações de contexto de acordo com tempo, localização, entre outros fatores.

Sistemas de recomendação geram sugestões baseadas em conjuntos de regras para coleções de itens recomendados a um grupo de usuários [Adomavicius e Tuzhilin 2005]. Uma série de estratégias podem ser utilizadas na definição de recomendações. Estas estratégias definem o tipo de sistema de recomendação. Elas podem ser: Filtragem colaborativa, Recomendação baseada em conteúdo, Recomendação baseada em contexto ou Abordagem híbrida [Adomavicius e Tuzhilin 2005] [Adomavicius e Tuzhilin 2011]. A Filtragem Colaborativa (Collaborative Filtering - CF) realiza análises do histórico de feedback dos usuários sobre recomendações previamente realizadas. Através desta análise, sistemas de recomendação podem explorar comportamentos parecidos entre usuários no processo de escolha e recomendação de itens aos usuários. Há uma série de técnicas de filtragem colaborativa que podem ser integradas entre si, como Neighborhood-Based Collaborative Filtering (NB), Item-Based Collaborative Filtering (IB), e Model-Based Collaborative Filtering (MB). 
A Filtragem baseada em conteúdo (Content-Based Recommendation - CBR) realiza análises das características dos itens que podem ser recomendados. Estas análises são geralmente feitas através da comparação de características entre coleções de itens. Recomendação baseada em contexto (Context-Based Recommendation - CxBR) utiliza análises de elementos de contexto informados no momento da recomendação. Os seguintes elementos podem ser considerados como elementos de contexto: Perfil do usuário, localização, dispositivo, entre outros. No processo de pré-filtragem de contexto (PreF), a filtragem baseada em contexto é realizada antes do processo de recomendação. No processo de pós-filtragem de contexto (PosF), o processo de recomendação é realizado, e o resultado posteriormente é filtrado de acordo com o contexto. Já no processo de recomendação com Modelagem de Contexto (Mod), a filtragem baseada em contexto e a recomendação são realizados no mesmo processo. Abordagens Híbridas (H) combinam mais de uma técnica de recomendação e têm sido aplicadas em trabalhos recentes [Hussein et al. 2014] [Quan e Cho 2014]. Esta combinação pode ser feita entre duas ou mais técnicas.

Sistemas Hipermídia Adaptativos (SHA) possuem uma característica que os diferencia dos outros sistemas: Eles utilizam modelagens explícitas de usuário, que representam informações de seu perfil, conhecimento do mesmo, e outras informações para auxiliar o sistema no processo de identificação do usuário, e posteriormente na adaptação do sistema em prol do usuário [Brusilovsky e Su 2002]. A adaptação de sistemas pode ser realizadas em diversos fatores. A Seleção de Conteúdo (Content Selecion - CS) está relacionada com a forma de apresentar o melhor conteúdo de acordo com o perfil de usuário e informações de contexto relacionadas ao usuário. Isto é definido por conjuntos de regras, que definem (i) a seleção de conceitos do domínio, (ii) a seleção de recursos, e (iii) geração de caminhos de aprendizagem apropriados aos usuários. A Apresentação de Conteúdo (Content Presentation - P) está relacionada a forma de como os conteúdos podem ser apresentados da melhor forma ao usuário baseado no perfil e em informações de contexto relacionadas ao usuário. A Apresentação e Suporte à Navegação (Navigation Support and Presentation - NPS) está relacionada ao uso de técnicas de adaptação para modificar a forma com que links são apresentados em conteúdos hipermídia, modificando a navegação de acordo com as informações sobre o perfil do usuário. Essas técnicas são utilizadas em diferentes áreas de aplicação, e na área educacional são interessantes visto sua capacidade de auxiliar individualmente cada aluno, ajudando-o na construção de seu conhecimento.

\section{Análise e Discussão}

Para obter uma visão mais clara sobre os artigos selecionados, agrupamos os artigos com base em suas palavras-chave. Elas foram escolhidas porque são geralmente definidas pelos autores e sumarizam em poucas palavras os tópicos de interesse do artigo. As palavras-chave foram extraídas de cada artigo e posteriormente analisadas. As que foram consideradas muito genéricas, como por exemplo algoritmos, teoria e experimentação, e as que apareceram somente uma vez foram removidas da base de dados.

Após a realização desta análise, foram obtidas 21 palavras-chave. As palavraschave resultantes são apresentadas na Figura 1(a). Após o término da filtragem, foram obtidas 18 palavras-chave utilizadas no processo de clusterização. O conjunto de artigos selecionados foi analisado mais uma vez com o objetivo de identificar quais palavraschave eram utilizadas em quais artigos, mesmo que a palavra não estivesse presente na seção de palavras-chave do artigo. A saída desta análise é um dataset que apresenta as 
relações entre cada artigo e as palavras-chave. O dataset está disponível para consulta em: http://bit.ly/1Msdyhu.

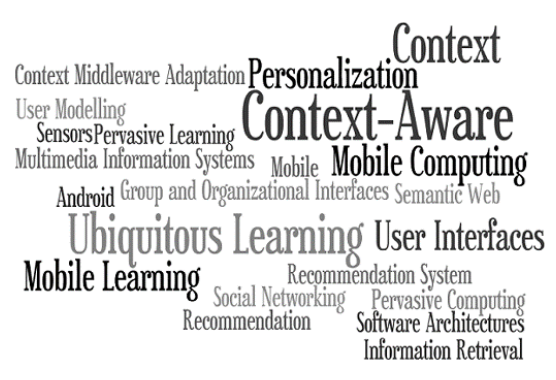

(a)

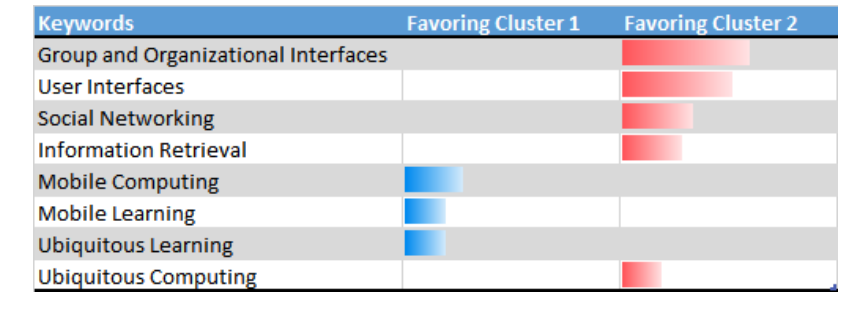

(b)

Figura 1. (a) Nuvem de palavras-chave dos artigos selecionados; (b) Atributos representativos em cada cluster

Para realizar a clusterização dos artigos, foi utilizado o algoritmo Microsoft Clustering, um algoritmo iterativo, que constrói modelos estatísticos da fonte de dados subjacente e naturalmente generaliza a bases de dados, que contém informações discretas e contínuas. Isto não acontece em algoritmos baseados em distância, como o K-Means [Tang et al. 2005]. O algoritmo primeiro cria clusters e depois calcula a probabilidade de um dado pertencer a um cluster em particular. Os resultados apresentados pelo algoritmo são apresentados na Figura 1(b). O algoritmo criou 2 clusters, com as 8 palavras chave que foram consideradas como estatisticamente relevantes. Os resultados foram ordenados pelo valor de probabilidade da palavra-chave pertencer ao cluster em que ela se encontra, do maior para o menor. O maior valor foi Group and Organizational Interfaces $(59,903)$ e o menor foi o da palavra-chave Ubiquitous Computing $(18,725)$.

A distribuição em percentual, de todos os atributos em cada cluster, é apresentada na Figura 2. Essa distribuição torna claro o motivo de que apenas oito atributos foram considerados representantes quando o algoritmo criou os clusters. Os dez atributos que não foram considerados como representantes são distribuídos em percentagens semelhantes, enquanto os outros oito atributos apresentam uma distribuição desigual entre os clusters, e assim, foram considerados representativos. O processo de clusterização contribuiu para mostrar um agrupamento entre os artigos da área. Ele mostrou que, em um lado, os artigos envolvidos com grupos e interfaces organizacionais estão trabalhando também com interfaces de usuário, redes sociais, recuperação de informação e computação ubíqua. Outro resultado interessante é que as palavras-chave Aprendizagem Ubíqua e Computação Ubíqua foram classificadas em clusters diferentes. Isto ocorreu porque, apesar da semelhança entre os termos, um deles foi considerado pelo algoritmo como mais representativo para um cluster que o outro. Porém, isto não significa que artigos que trabalham com aprendizagem ubíqua não trabalhem com Computação Ubíqua ou o contrário. Uma constatação interessante foi que os artigos que trabalham com técnicas de adaptação têm pouca relação com técnicas de recomendação. Além disso, foi constatado que em nosso conjunto de dados apenas três artigos estão diretamente relacionados com os quatro temas de interesse deste artigo, sendo todos artigos do tipo survey. No entanto estes artigos tendem a se concentrar na recomendação ou em abordagens adaptativas. Não foi encontrado nenhum trabalho que apresente uma abordagem de fusão das contribuições de ambas as áreas. Este fato ressalta que a integração entre as técnicas de recomendação e de adaptação é um tema a ser explorado. 


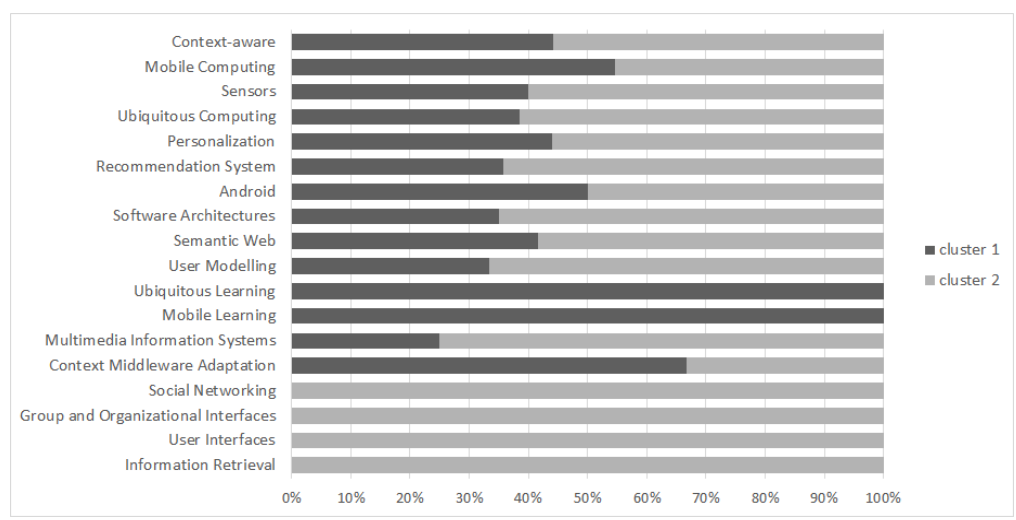

Figura 2. Distribuição de palavras-chave em cada cluster

Tal fato responde a nossa pergunta de pesquisa, apontando que, não existem trabalhos que realizam recomendação e utilizam sensibilidade ao contexto e adaptação no ambiente ubíquo, os trabalhos tendem a concentrar suas contribuições em apenas uma das áreas. Não foi encontrada uma abordagem mostrando uma integração precisa das técnicas, suas vantagens, desvantagens ou até mesmo uma comparação entre uma abordagem de adaptação, recomendação e uma abordagem clássica. Com o intuito de confirmar os resultados encontrados através da análise das palavras-chave foi realizado ainda outro estudo sobre os artigos. Nesta nova análise cada trabalho foi categorizado segundo os conceitos definidos em cada domínio a que se relaciona este mapeamento, tais conceitos foram apresentados nas Seções 3, 4 e 5. A Tabela 2 apresenta os artigos categorizados segundo tais conceitos.

Na Tabela 2 são elencados 40 artigos, três artigos selecionados anteriormente após a $2^{\mathrm{a}}$ filtragem de trabalhos não foram considerados durante a categorização pelo fato dos artigos serem do tipo survey, que tratam dos conceitos e técnicas relacionados aos domínios de pesquisa, porém não têm foco na utilização de algum desses conceitos em uma aplicação ou abordagem proposta. Tais artigos são os trabalhos 3, 30 e 43. Os demais artigos foram classificados em três tipos: Artigos publicados em Workshops (WSP), artigos publicados em conferências (CNF), e artigos publicados em periódicos $(\mathbf{J N})$. Cada artigo foi analisado segundo a técnica utilizada nos domínios de sensibilidade ao contexto, sistemas de recomendação e sistemas adaptativos. No domínio de sensibilidade ao contexto, além do tipo de modelagem e do nível do contexto, cada artigo foi categorizado segundo a utilização feita da informação de contexto que pode ser: Consulta de dados (DQ), adaptação de serviços (AS), adaptação de interfaces (IA), e uso de recursos (UR). No domínio de sistemas de recomendação os artigos foram categorizados segundo a abordagem de recomendação empregada (classificação) e a técnica empregada na abordagem (característica). Por fim, a categorização de artigos no domínio de sistemas adaptativos levou em consideração o fator de adaptação e a técnica utilizada no fator.

Os trabalhos que não apresentaram algum dos conceitos verificados tiveram suas células preenchidas com 2 hifens (--). Já se o trabalho apresentou um conceito não coberto neste mapeamento, sua célula foi preenchida com 2 asteriscos $(* *)$. A análise da Tabela 2 mostra que nenhum dos trabalhos verificados apresenta uma abordagem de recomendação que também seja adaptativa, confirmando os resultados encontrados através da análise das palavras-chave dos artigos. $\mathrm{O}$ único trabalho que apresentou um fator (estilo de navegação - NPS) e uma técnica de adaptação (baseado em preferências - PB) não trata de recomendação. Outras constatações, referentes ao domínio de sensibilidade ao contexto, são que poucos trabalhos explicitam seus modelos de contexto ou dizem como eles 
foram elaborados, metade dos trabalhos utiliza a informação contextual em alto nível (HL) e a utilização mais comum da informação é para fazer consulta a dados (DQ) sendo que tais consultas por vezes têm objetivo também de adaptar interface ou serviços.

Tabela 2. Análise de trabalhos de acordo com os conceitos de cada domínio

\begin{tabular}{|c|c|c|c|c|c|c|c|c|}
\hline \multicolumn{2}{|c|}{ Informações Gerais } & \multicolumn{3}{|c|}{ Sensibilidade ao Contexto } & \multicolumn{2}{|c|}{ Sistemas de Recomendação } & \multicolumn{2}{|c|}{ Sistemas Adaptativos } \\
\hline 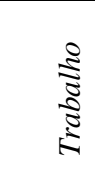 & 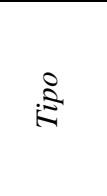 & 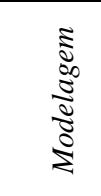 & $\frac{\widetilde{\Xi}}{\dot{z}}$ & 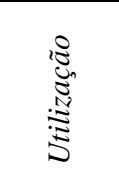 & 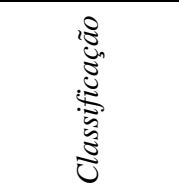 & 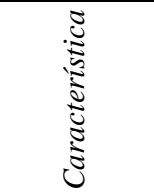 & $\underset{\mathbf{D}}{\overline{0}}$ & 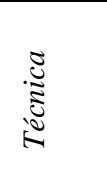 \\
\hline 1 & WSP & $O$ & $H L$ & $A S$ & -- & -- & -- & -- \\
\hline 2 & $\mathrm{CNF}$ & $O$ & $I L$ & $U R$ & $C F$ & $N B$ & -- & -- \\
\hline 4 & $\mathrm{JN}$ & -- & $I L$ & $U R$ & $H$ & $N B+M o d$ & -- & -- \\
\hline 5 & $\mathrm{CNF}$ & -- & $I L$ & $I A+U R$ & $C X B R$ & PreF & -- & -- \\
\hline 6 & $\mathrm{CNF}$ & -- & $L L$ & $I A+D Q$ & $C x B R$ & PreF & -- & -- \\
\hline 7 & $\mathrm{CNF}$ & -- & $L L$ & $D Q$ & $C x B R$ & PreF & -- & -- \\
\hline 8 & $\mathrm{CNF}$ & $G M$ & $H L$ & $A S$ & $H$ & $I B+M o d$ & -- & -- \\
\hline 9 & $\mathrm{CNF}$ & -- & $L L$ & $U R$ & $H$ & $N B$ & -- & -- \\
\hline 10 & WSP & -- & $L L$ & $U R$ & $H$ & $N B$ & -- & -- \\
\hline 11 & $\mathrm{JN}$ & $O$ & $H L$ & $D Q$ & $C F$ & $N B$ & -- & -- \\
\hline 12 & WSP & -- & $L L$ & $I A+D Q$ & $H$ & $N B$ & -- & -- \\
\hline 13 & $\mathrm{CNF}$ & $L B$ & $I L$ & $I A$ & -- & -- & -- & -- \\
\hline 14 & $\mathrm{JN}$ & -- & -- & -- & $* *$ & $* *$ & -- & -- \\
\hline 15 & $\mathrm{CNF}$ & -- & $H L$ & $U R$ & -- & -- & -- & -- \\
\hline 16 & $\mathrm{CNF}$ & -- & $L L$ & $A S$ & $C x B R$ & $* *$ & -- & -- \\
\hline 17 & WSP & $L B$ & $H L$ & $A S$ & -- & -- & -- & -- \\
\hline 18 & $\mathrm{CNF}$ & -- & $H L$ & $U R$ & $C F$ & $N B$ & -- & -- \\
\hline 19 & $\mathrm{CNF}$ & -- & $I L$ & $A S+I A$ & $C x B R$ & $* *$ & -- & -- \\
\hline 20 & JN & -- & $H L$ & $U R$ & -- & -- & -- & -- \\
\hline 21 & $\mathrm{CNF}$ & -- & $H L$ & $A S$ & $C x B R$ & $* *$ & -- & -- \\
\hline 22 & $\mathrm{CNF}$ & -- & $H L$ & $A S$ & $H$ & PreF $+N B$ & -- & -- \\
\hline 23 & $\mathrm{CNF}$ & -- & $H L$ & $A S$ & $H$ & $\mathrm{PreF}+\mathrm{NB}$ & -- & -- \\
\hline 24 & $\mathrm{CNF}$ & -- & $L L$ & $D Q$ & $C X B R$ & $* *$ & -- & -- \\
\hline 25 & WSP & -- & $H L$ & $U R$ & $C x B R$ & $* *$ & -- & -- \\
\hline 26 & WSP & $O$ & $L L$ & $D Q+A S$ & $C x B R$ & PreF & -- & -- \\
\hline 27 & $\mathrm{CNF}$ & -- & $H L$ & $D Q+A S$ & $H$ & $I B+M o d$ & -- & -- \\
\hline 28 & $\mathrm{JN}$ & $K V$ & $L L$ & $D Q+A S$ & $C X B R$ & -- & -- & -- \\
\hline 29 & $\mathrm{JN}$ & -- & $L L$ & $D Q$ & $C F$ & $I B$ & -- & -- \\
\hline 31 & $\mathrm{JN}$ & $O$ & $H L$ & $D Q$ & $C x B R$ & $M B$ & -- & -- \\
\hline 32 & $\mathrm{JN}$ & -- & -- & -- & $H$ & $N B$ & -- & -- \\
\hline 33 & $\mathrm{JN}$ & $G M$ & $H L$ & $D Q$ & $H$ & $N B$ & -- & -- \\
\hline 34 & $\mathrm{JN}$ & $G M$ & $H L$ & $D Q$ & $C x B R+C B R$ & $I B$ & -- & -- \\
\hline 35 & $\mathrm{JN}$ & -- & -- & -- & $H$ & $I B$ & -- & -- \\
\hline 36 & $\mathrm{JN}$ & $O$ & $H L$ & $D Q+I A$ & $H$ & $I B$ & -- & -- \\
\hline 37 & $\mathrm{JN}$ & -- & -- & -- & $C B R$ & $I B$ & -- & -- \\
\hline 38 & $\mathrm{JN}$ & $G M$ & $H L$ & $I A$ & -- & -- & $N P S$ & $P B$ \\
\hline 39 & $\mathrm{CNF}$ & -- & -- & -- & $C B R$ & $I B$ & -- & -- \\
\hline 40 & $\mathrm{JN}$ & $O$ & $H L$ & $D Q$ & $C x B R$ & $I B$ & -- & -- \\
\hline 41 & $\mathrm{JN}$ & $K V$ & $L L$ & $* *$ & -- & -- & -- & -- \\
\hline 42 & CNF & $G M$ & $H L$ & $D Q$ & -- & -- & -- & -- \\
\hline
\end{tabular}


No domínio de sistemas de recomendação as abordagens mais utilizadas são recomendações sensíveis ao contexto $(\mathbf{C x B R})$ e recomendações híbridas $(\mathbf{H})$, com 13 e 12 ocorrências respectivamente e a técnica mais utilizada é baseada em vizinhança (NB). Diante deste cenário, pode-se afirmar novamente que não foram encontrados trabalhos que propõem a utilização de técnicas de adaptação a sistemas de recomendação.

\section{Conclusões}

Este trabalho apresenta um mapeamento sistemático de abordagens de recomendação utilizadas em ambientes ubíquos tradicionais e educacionais, sendo tais abordagens sensíveis ao contexto e adaptativas. A inclusão de abordagens tradicionais ao mapeamento, tem o intuito de abordar a maior quantidade de trabalhos possíveis e descobrir possíveis oportunidades de pesquisa. A busca por artigos foi executada em quatro motores de busca diferentes e após duas filtragens, 43 artigos foram selecionados para análise. Tais trabalhos foram então clusterizados com base em suas palavras-chave e tal processo mostrou que não existem abordagens de recomendação que são também adaptativas.

Outrossim, apesar de serem encontrados alguns trabalhos de abordagens de recomendação para recursos do ambiente, dificilmente são encontrados sistemas adaptativos que levam em consideração o ambiente e os recursos próximos ao usuário. Da mesma maneira, sistemas de recomendação geralmente não se preocupam em recomendar o melhor caminho de uso dos itens recomendados ou não se preocupam com a apresentação de tais recursos, importantes tarefas ao se tratar de conteúdo educacional. O processo de clusterização e a análise manual dos artigos, sumarizada na Tabela 2, foram de fundamental importância pois permitiram o agrupamento dos trabalhos através de suas similaridades e desta maneira foi possível extrair conclusões que permitiu responder à questão de pesquisa. Como trabalhos futuros iremos nos aprofundar nos trabalhos levantados, de maneira a considerar também as referências utilizadas em cada trabalho. Com isso, espera-se entender o início e evolução das áreas de pesquisa e o relacionamento entre elas. Além disso, será executado um processo de clusterização sobre os resumos dos trabalhos com o objetivo de encontrar mais atributos e características interessantes aos clusters.

\section{Referências Bibliográficas}

Adomavicius, G. and Tuzhilin, A. (2005). Toward the next generation of recommender systems: a survey of the state-of-the-art and possible extensions. IEEE Transactions on Knowledge and Data Engineering, v. 17, n. 6, p. 734-749.

Adomavicius, G. and Tuzhilin, A. (2011). Context-Aware Recommender Systems. In: Ricci, F.; Rokach, L.; Shapira, B.; Kantor, P. B.[Eds.]. . Recommender Systems Handbook SE - 7. Springer US. p. 217-253.

Bettini, C., Brdiczka, O., Henricksen, K., et al. (2010). A survey of context modelling and reasoning techniques. Pervasive and Mobile Computing, v. 6, n. 2, p. 161-180.

Bouzeghoub, A., Do, K. N. and Wives, L. K. (2009). Situation-Aware Adaptive Recommendation to Assist Mobile Users in a Campus Environment. In 2009 Int. Conf. on Advanced Information Networking and Applications., AINA '09. IEEE.

Brusilovsky, P. (2001). Adaptive Hypermedia. User Modeling and User-Adapted Interaction, v. 11, n. 1-2, p. 87-110. 
Brusilovsky, P. and Su, H. (2002). Adaptive Visualization Component of a Distributed Web-Based Adaptive Educational System. Lecture Notes in Computer Science, v. 2363, p. 229-238.

Dey, A. K. (2001). Understanding and Using Context. Personal and Ubiquitous Computing, v. 5, n. 1, p. 4-7.

Gasparini, I., De Oliveira, J. P. M., Pimenta, M. S., et al. (2009a). AdaptWeb®Evolução e Desafios. Cadernos de Informática, v. 4, n. 2, p. 47-56.

Gasparini, I., Lichtnow, D., Pimenta, M. S. and Oliveira, J. P. M. De (nov 2009). Quality Ontology for Recommendation in an Adaptive Educational System. In 2009 Int. Conf. on Intelligent Networking and Collaborative Systems. IEEE.

Hussein, T., Linder, T., Gaulke, W. and Ziegler, J. (2014). Hybreed: A software framework for developing context-aware hybrid recommender systems. User Modeling and User-Adapted Interaction, v. 24, n. 1-2, p. 121-174.

Jones, V. and Jo, J. H. (2004). Ubiquitous learning environment: An adaptive teaching system using ubiquitous technology. In Beyond the comfort zone: Proceedings of the 21st ASCILITE Conference.

Kitchenham, B. A. and Charters, S. (2007). Guidelines for performing Systematic Literature Reviews in Software Engineering.

Makris, P., Skoutas, D. N. and Skianis, C. (2013). A survey on context-aware mobile and wireless networking: On networking and computing environments' integration. Communications Surveys \& Tutorials, IEEE, v. 15, n. 1, p. 362-386.

Maran, V., Augustin, I. and Oliveira, J. P. M. De (2014). Are The Integrations Between Ontologies and Databases Really Opening the Closed World in Ubiquitous Computing? In Int. Conf. on Software Engineering \& Knowledge Engineering.

Medeiros Machado, G. and De Oliveira, J. (2014). Context-aware adaptive recommendation of resources for mobile users in a university campus. In Wireless and Mobile Computing, Networking and Communications (WiMob), 2014 IEEE

Petersen, K., Feldt, R., Mujtaba, S. and Mattsson, M. (2008). Systematic Mapping Studies in Software Engineering. In Proceedings of the 12th Int. Conf. on Evaluation and Assessment in Software Engineering. , EASE'08. British Computer Society.

Quan, J.-C. and Cho, S.-B. (2014). A Hybrid Recommender System Based on AHP That Awares Contexts with Bayesian Networks for Smart TV. Hybrid Artificial Intelligence Systems. Springer. p. 527-536.

Riboni, D. and Bettini, C. (2012). Private context-aware recommendation of points of interest: An initial investigation. In Pervasive Computing and Communications Workshops (PERCOM Workshops), 2012 IEEE Internaional Conference on.

Ricci, F., Rokach, L. and Shapira, B. (2011). Introduction to Recommender Systems Handbook. In: Ricci, F.; Rokach, L.; Shapira, B.; Kantor, P. B.[Eds.]. Recommender Systems Handbook SE - 1. Springer US. p. 1-35.

Tang, Z., Maclennan, J. and Kim, P. P. (2005). Building data mining solutions with OLE DB for DM and XML for analysis. ACM SIGMOD Record. ACM.

Weiser, M. (1991). The computer for the 21st century. Scientific American, p. 94-104. 